virtually no spontaneous activity, and needed prompting to attend to all aspects of her personal care. Her mood appeared flat but she denied any feelings of depression, pessimism or hopelessness, slept and ate normally, and there were no other signs suggestive of depression. Her mental state showed no improvement in the hospital environment over a 6-week period prior to treatment.

She had a previous history of hyperthyroidism and partial thyroidectomy many years earlier. Biochemical testing showed total $\mathrm{T}_{4} 131 \mathrm{nmol} / \mathrm{h}$ (normal range 60-170), free $T_{4} 17 \mathrm{nmol} / 1(9-22)$, free $T_{3}$ $8.1 \mathrm{pmol} / \mathrm{l}$ (4.6-8.2), and TSH $0.05 \mathrm{mu} / \mathrm{l}(0.4-5)$. A thyroid scintigram and ultrasound showed an active nodule in the thyroid remnant with suppression of activity in the remaining thyroid tissue. In view of the marked suppression of TSH a diagnosis of subclinical hyperthyroidism was made and she was treated with carbimazole $15 \mathrm{mg}$ b.d. Steady improvement in her mental state was seen over the next 3 weeks, with increased physical activity, increased social and emotional responsiveness, and a subjective report of feeling healthier and more energetic. She proved able to care for herself once again, was discharged from hospital and has remained well since.

Sawin, C. T., Gell.er, A., Wolf, P. A., el al (1994) Low serum thyrotropin concentrations as a risk factor for atrial fibrillation in older persons. New' England Journal of Medicine, 331, 1249 1252

Schlote, B., Nowotny, B., SchaAf, L., et al (1992) Subclinical hyperthyroidism: physical and mental state of patients. European Archives of Psychiatry and Clinical Neuroscience, 241, 357-364.

M. J. EALES

Avalon Somerset NHS Trust

P. VAN DER MERWE

Somerset TA6 $3 L S$

\section{Antidepressants in pregnancy and breastfeeding}

SIR: We were interested to read the letter by Kent \& Laidlaw (1995) about withdrawal symptoms in a baby who had been breastfed by a mother taking sertraline.

We report a 32-year-old woman who was started on sertraline $150 \mathrm{mg}$ daily when 20 weeks pregnant. She delivered a healthy baby at term and breastfed for 11 days while on the same dose. There was no behavioural change in the baby after cessation of breastfeeding. Perhaps the appearance of withdrawal symptoms is dose related in that our patient was taking sertraline $150 \mathrm{mg}$ daily while Kent \& Laidlaw's patient was on $200 \mathrm{mg}$.
In the absence of controlled studies on the use of most antidepressants in pregnancy and breastfeeding, it would be useful if there were a register of cases where an antidepressant had been used and of the outcome. The setting up of such a register is under consideration by the Marce Society: International Association for Psychiatric Disorders of Childbearing.

KENT, L. S. W. \& Laidlaw, J. D. D. (1995) Suspected congenital sertraline dependence (letter). British Journal of Psychiatry, 167, $412-413$

D. A. Ratan

Leicestershire Mental Health Service NHS Trust

Leicester LE5 4PW

T. FRIEDMAN

Marce Society

Leicester General Hospital

Leicester LE5 4PW

Neuropsychological function in manic-depressive psychosis

SIR: McKay et al's study (1995) requires comment. It does not appear that the three groups described by the authors are entirely comparable. The impaired group had 9 out of the 10 cases with bipolar affective disorder, whereas the ratio for the younger group was 2 out of 22 and for the elderly group 1 out of 11. If comparability is assumed then an assumption also needs to be made that the underlying disease process in bipolar affective disorder and major depression is identical. This is important because impairment was predominantly a feature of the bipolar group.

If cognitive impairment is a feature of the severe and chronic state, then it is also reasonable to speculate that a spectrum of neuropsychological deficit exists that increases with increasing chronicity and severity, although no such evidence was found in this study. Incidentally neither chronicity nor severity were defined. Presumably chronicity was determined on the basis of the number of years of illness but severity is harder to understand. Was it on the basis of number of symptoms, intensity of symptoms, or the level of care required? Hopefully selection was based on identical criteria.

While the authors dismiss drug effects by citing studies that report on the effect of drugs on cognitive function while the drugs are used individually, in clinical practice that is not the case. Chronicity and severity easily translates as intractability, implying that drugs are used in combination, at more than the usual doses and for prolonged periods of 